\title{
Multilayer Structures with Giant Magnetoresistance
}

\author{
F. Stobiecki ${ }^{a}$ And T. Stobiecki ${ }^{b, *}$ \\ ${ }^{a}$ Institute of Molecular Physics, Polish Academy of Sciences \\ Smoluchowskiego 17, 60-179 Poznań, Poland \\ ${ }^{b}$ Department of Electronics, University of Mining and Metallurgy
}

Al. Mickiewicza 30, 30-059 Kraków, Poland

The phenomenological description of the giant magnetoresistance effect as well as the discussion of the requirements which must be fulfilled in giant magnetoresistance thin film structures are given in the first part of our review. In the second part the magnetization reversal and giant magnetoresistance effect of antiferromagnetically coupled multilayers, spin valve and pseudo-spin valve thin film structures are explained. For these structures we also discuss the influence of the structure defects such as surface roughness and pinholes on the giant magnetoresistance effect.

PACS numbers: 75.70.Pa, 75.70.-i

\section{Introduction}

Magnetoresistance (MR) is the change in electrical resistance of a conductor caused by magnetic field. The dominating origin of this effect can be different dependently on the material and/or the structure of the sample. In nonmagnetic conductors the MR is due to the Lorentz force the magnetic field exerts on moving electrons. This effect is relatively small, for example in $\mathrm{Cu}$ the relative increase in resistance with magnetic field is $\Delta R /(R H)=1.3 \times 10^{-3} \% / \mathrm{kOe}$ (at room temperature, RT) [1]. In magnetic materials and particularly in magnetic thin film structures the spin polarization of electrons generates another, usually larger, contribution to the MR effect. In ferromagnetic samples the resistance depends on the mutual orientation of the magnetization and the current directions. About thirty years ago this effect known in literature as anisotropic magnetoresistance (AMR) was intensively investigated in thin ferromagnetic films. In permalloy $\left(\mathrm{Ni}_{80} \mathrm{Fe}_{20}\right)$ films the resistance changes up to $5 \%$ were observed in magnetic field

* corresponding author; e-mail: stobieck@uci.agh.edu.pl 
$H \leq 10$ Oe [2-5]. Such considerable resistance changes at low magnetic field (high field sensitivity) make AMR effect attractive for various applications (see for example $[6,7]$ ). From all of them, the magnetoresistive read heads for magnetic hard discs, introduced on the market by IBM in 1990, seem to be particularly important. Thus AMR effect was the precursor for application of new MR effects such as giant magnetoresistance (GMR), tunnel magnetoresistance (TMR) [8] and colossal magnetoresistance (CMR) [9].

In this lecture we concentrate on the giant magnetoresistance effect in current in plane (CIP) configuration only. Particularly, in Sec. 2 the origin of GMR effect is explained, in Sec. 3 different GMR thin film structures are described.

\section{Giant magnetoresistance effect - phenomenological description}

The GMR effect $(\Delta R / R \approx 70 \%$ at $4.2 \mathrm{~K})$ was discovered in $\mathrm{Fe} / \mathrm{Cr}$ multilayers (MLs) [10, 11]. Two years earlier [12, 13] it was demonstrated that for such MLs due to exchange interlayer coupling (see Sec. 3.1) between ferromagnetic layers (Fe in this case) across a metallic spacer layer (Cr) the antiparallel magnetization configuration (antiferromagnetic interlayer exchange coupling) between neighbouring Fe layers can be obtained for a given thickness of Cr. It should be noticed that GMR effect described in Ref. [10,11] was observed only for Fe/Cr MLs with antiferromagnetic coupling. For such MLs the resistance drops as the magnetization configuration in neighbouring Fe layers goes from antiparallel alignment at magnetic field $H=0$, to parallel one at $H=H_{\mathrm{S}}\left(H_{\mathrm{S}}\right.$ is the saturation field, i.e. $H$ necessary to order the magnetization of ferromagnetic layers in field direction). The common explanation of the GMR effect (see e.g. [14]) is the different spin-dependent scattering probability for spin-up and spin-down electrons, i.e., different resistivity for either spins configuration $\left(\rho_{\uparrow}\right.$ and $\left.\rho_{\downarrow}\right)$. Due to the Pauli exclusion principle, electrons can be scattered from impurities or defects into the quantum states in the vicinity of the Fermi level $\left(E_{\mathrm{F}}\right)$. Thus the scattering probability is proportional to the number of states available for scattering at $E_{\mathrm{F}}$, i.e., to the density of states $\left(\rho \propto D\left(E_{\mathrm{F}}\right)\right)$. In the transition metal ferromagnet, the up and down spin bands are split and the density of states at the Fermi level for electrons with spin up and down is different $\left(D_{\uparrow}\left(E_{\mathrm{F}}\right) \neq D_{\downarrow}\left(E_{\mathrm{F}}\right)\right.$ ) (Fig. 1). For majority electrons with spin parallel to the local magnetization (spin up) usually the density of states at the Fermi level is lower as for minority electrons with spin oriented antiparallel (spin down) $D_{\uparrow}\left(E_{\mathrm{F}}\right)>D_{\downarrow}\left(E_{\mathrm{F}}\right)$ and, consequently, $\rho_{\uparrow}<\rho_{\downarrow}$. Considering low probability of electron scattering with spin reorientation we can assume that the total current can be viewed as the one consisting of two channels in parallel (Mott's two-current model [15]), one for electrons with spin up and the other one with spin down. Taking into account two-current model and the difference in resistivity $\left(\rho_{\uparrow}<\rho_{\lrcorner}\right)$we can explain the GMR effect in layered structures consisting of ferromagnetic (F) layers separated by non-ferromagnetic metallic layer $(\mathrm{S})$. 

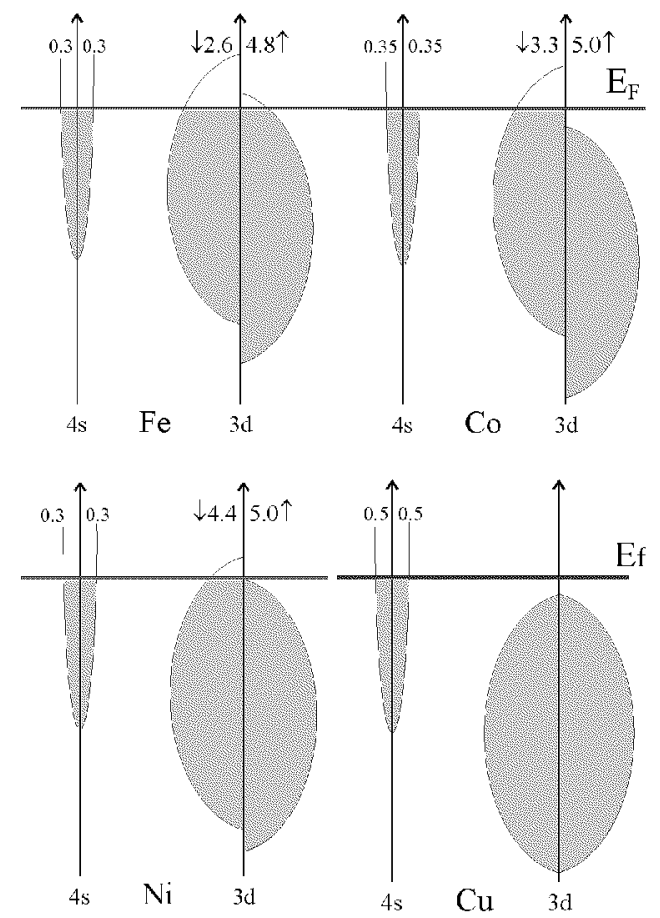

Fig. 1. Schematic spin-split density of states (DOS) for $3 d$ transition metal (Fe, Co and $\mathrm{Ni}$ ) and $\mathrm{Cu}$ representing the spacer and magnetic layer, respectively. The relative positions of the bands for spin-up and spin-down electrons give rise to spin-dependent scattering.

For parallel magnetization configuration ( $\uparrow$ ) of adjacent F layers (Fig. 2a) there are different scattering probabilities for either spin directions. The electrons with spin up (low scattering probability) form low resistivity channel and the total resistivity $\rho_{\uparrow \uparrow}=\rho_{\uparrow} \rho_{\downarrow} /\left(\rho_{\uparrow}+\rho_{\downarrow}\right)$ is low. For antiparallel arrangement (Fig. 2b) of magnetization in successive $\mathbf{F}$ layer there are similar scattering events for both types of electrons. What is the low resistivity electron species in a layer becomes the high resistivity electron species in the next. Thus in the channel for electrons with spin up as well as with spin down the resistivity is expressed as $\left(\rho_{\uparrow}+\rho_{\downarrow}\right) / 2$ and the total resistivity $\rho_{\uparrow \downarrow}=\left(\rho_{\uparrow}+\rho_{\downarrow}\right) / 4$. Considering the equations which determine resistivities for both magnetization configuration we can express the resistance changes observed during magnetization reorientation from antiparallel to parallel as

$$
\begin{aligned}
\mathrm{GMR} & =\Delta R / R=\left(R_{\uparrow \downarrow}-R_{\uparrow \uparrow}\right) / R_{\uparrow \uparrow} \\
& =\left[\left(\rho_{\downarrow}-\rho_{\uparrow}\right) /\left(\rho_{\downarrow}+\rho_{\uparrow}\right)\right]^{2}=[(\alpha-1) /(\alpha+1)]^{2} .
\end{aligned}
$$

In the above equation the parameter $\alpha=\rho_{\downarrow} / \rho_{\uparrow}$ describes the difference in the scattering processes occurring in the volume of ferromagnetic layers for both spin 


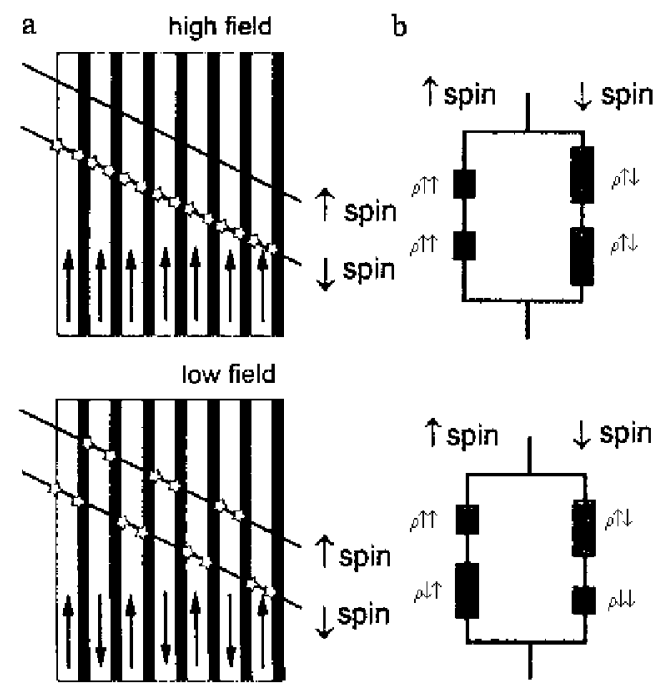

Fig. 2. (a) Schematic explanation of GMR in a magnetic multilayers; arrows in the layers indicate the magnetization direction; $\uparrow \uparrow$ ferromagnetic and $\uparrow \downarrow$ antiferromagnetic state; stars represent electron scattering at the interfaces. (b) The low resistivity state is for electrons with spin parallel to the local magnetization, when the high resistivity is for electrons with antiparallel spin to the magnetization.

orientations. However, due to higher concentration of scattering centres (impurities and defects) at the interface of thin film layered structures, the scattering of electrons in interface region gives usually the main contribution to the GMR effect. Although spin dependent scattering in the volume and/or at the interfaces explains the microscopic origin of the GMR effect, in macroscopic samples two supplementary requirements must be fulfilled. Firstly, there must exist a certain non-collinear configuration of magnetization in adjacent ferromagnetic layers which can be changed by an external magnetic field.

The resistance changes in $\mathrm{F}_{1} / \mathrm{S} / \mathrm{F}_{2}$ structures vs. the angle $\varphi$ between magnetization directions of $\mathrm{F}_{1}$ and $\mathrm{F}_{2}$ layers are expressed as [16]:

$$
R(\varphi)=R_{0}+\Delta R(1-\cos \varphi) / 2 .
$$

Thus the GMR effect is maximal for structures in which the changes of $\varphi$ with magnetic field are from $180^{\circ}$ to $0^{\circ}$ (from antiparallel to parallel configuration).

The second requirement concerns the relation between the thickness of individual layers and mean free path (MFP) (typically $10 \mathrm{~nm}$ for metallic films) of spin-up and spin-down electrons. The GMR will exist only if electrons can sample more than one ferromagnetic layer. Thus with increasing thickness of $\mathrm{S}$ layer $\left(t_{\mathrm{S}}\right)$ in $\mathrm{F} / \mathrm{S} / \mathrm{F}$ structures the GMR effect decreases and for $t_{\mathrm{S}} \geq$ MPF disappears. This effect, called in the literature as shunting effect, explains also the decrease in GMR with increasing thickness of ferromagnetic layers $\left(t_{\mathrm{F}}\right)$ in which the interface scattering is more efficient than in bulk. However, both for $\mathrm{F}$ and $\mathrm{S}$ layers the minimal 
thickness assuring suitable GMR is also limited. The layer by layer (Frank-van der Merve) growth mode of thin films is realized only for well defined deposition conditions. Volmer-Weber or Stranski-Krastanow growth mode is observed more frequently. In these cases the continuous layer (full covering of the deposited area) is formed for nominal layer thickness essentially higher than one monolayer. The discontinuities of S layer in F/S/F structures, i.e., the existence of magnetic bridges between ferromagnetic layers can destroy antiparallel alignment of magnetization and thus GMR effect (see e.g. [17-20]). The granular structure of $\mathrm{F}$ layers for small clusters take one superparamagnetic behaviour [21]. Therefore the optimal thickness range both for $\mathrm{S}$ and $\mathrm{F}$ layers in layered GMR structures is strongly limited (neglecting non-typical structures $0.4 \leq t_{\mathrm{F}} \leq 3 \mathrm{~nm}, 0.8 \leq t_{\mathrm{S}} \leq 3 \mathrm{~nm}$ ). Of course for such small thicknesses the influence of structural defects (diffuse and/or rough interface, discontinuity of spacer layer) on magnetic and magnetoresistance properties can be very strong.

\section{Thin film structures with GMR}

Due to possible application as magnetic field detectors and particularly as magnetic read heads, a large number of thin film structures have been investigated during last years. It was demonstrated that the GMR effect is present not only in magnetic multilayers but also in a number of other artificial structures in which the magnetoresistance results from ordering of magnetization configurations due to the magnetic field. In this section we shall discuss basic systems as: periodic multilayers, spin valves and pseudo-spin valves in which GMR effect is observed.

\subsection{Periodic multilayers with antiferromagnetic exchange coupling}

As was mentioned above the periodic multilayers $\mathrm{F} /(\mathrm{S} / \mathrm{F})_{N}$ (where $N$ is repetition number of bilayer $\mathrm{S} / \mathrm{F}$ ) were historically first system in which GMR was observed. Antiparallel alignment of magnetization in neighbouring ferromagnetic sublayers is due to interlayer exchange coupling (IEC) via conduction electrons of non-ferromagnetic spacer S. Many review articles have been written on this subject where theory and first experiments were discussed (see e.g. [14, 22-28]). Therefore in this paper we confine ourselves to mentioning only the behaviour of IEC which are important to explain GMR in periodic MLs:

- The oscillation of the coupling energy $J$ (ferromagnetic $J>0$ and $\uparrow \uparrow$, antiferromagnetic coupling $J<0$ and $\uparrow \downarrow$ ) and decay of the amplitude oscillations with increase in the thickness spacer $\left(t_{\mathrm{S}}\right)$. The perpendicular configuration of magnetizations in the special conditions were also observed as a result of IEC.

- The oscillation period of IEC depends on band and crystal structure of the material of the spacer. For polycrystalline MLs the typical values of the period are equal to several lattice constants. 
- The coupling energy $(J)$ of IEC depends also on the type of materials of the ferromagnetic and spacer layers. The strongest coupling is observed for such kind of materials which are characterized by high contrast in the spin dependent reflectivity. This condition is fulfilled if material of ferromagnetic and spacer layer is selected from the same or neighbouring column of the periodic system. The imperfections of the interface structure between ferromagnetic and spacer layer (e.g. roughness and/or not sharp concentration gradient) and crystal structure of the spacer (e.g. amorphous) decreases the $J$ value.

- For MLs with negligible anisotropy and thickness of the spacer $\left(t_{\mathrm{S}}\right)$ assuring antiferromagnetic coupling, the coupling energy $(J)$ is determined by saturation field $H_{\mathrm{S}}$ (field necessary to order the magnetization vectors, of each individual ferromagnetic layers, in the field direction) by the relation $J_{\mathrm{AF}}=-M_{\mathrm{S}} H_{\mathrm{S}} t_{\mathrm{F}} / 4$ (where $M_{\mathrm{S}}$ is the saturation magnetization and $t_{\mathrm{F}}$ is the thickness of ferromagnetic layers, respectively).

As we have mentioned in Sec. 2 the main requirements for GMR effect are the changes of mutual magnetization configurations under external magnetic field. In

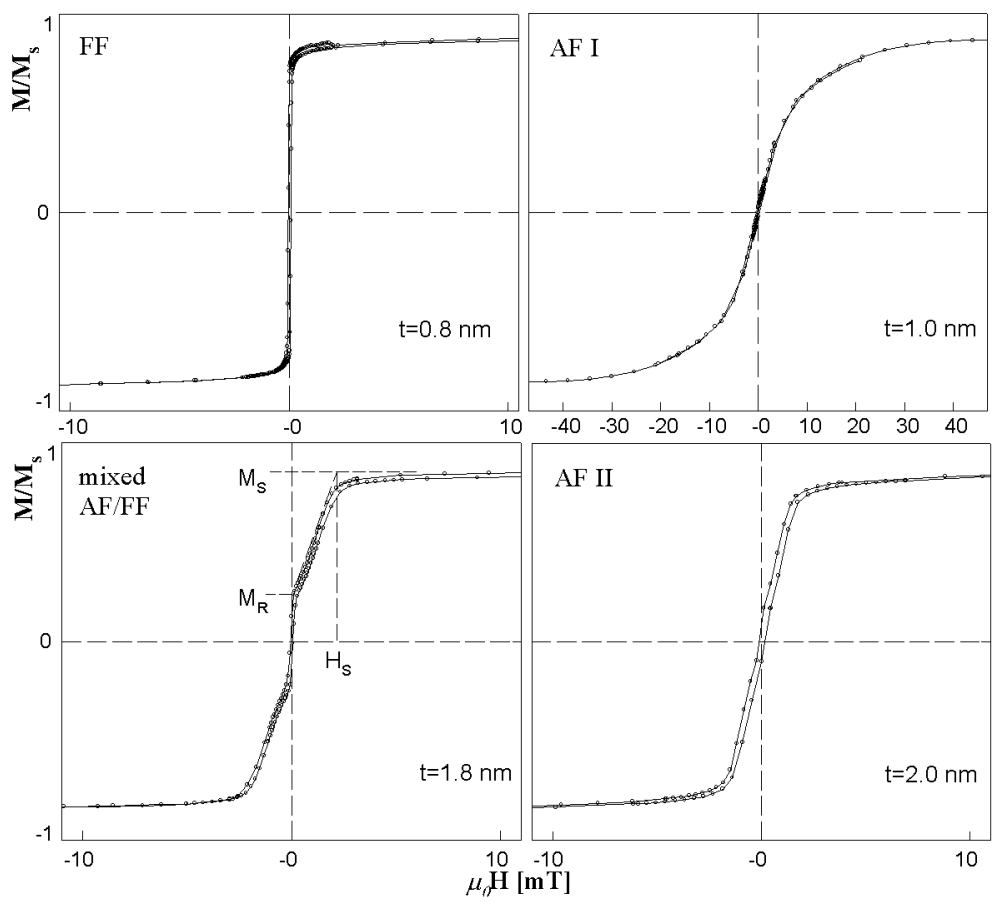

Fig. 3. Magnetization hysteresis loop of $\mathrm{Ni}_{83} \mathrm{Fe}_{17}(2 \mathrm{~nm}) / \mathrm{Cu}(t)$ multilayers for different thicknesses of $\mathrm{Cu}$ spacer. The coupling energy of the first and second antiferromagnetic maximum are $J_{\mathrm{AF} \mathrm{I}}=-4.6 \times 10^{-6} \mathrm{~J} / \mathrm{m}^{2}$ and $J_{\mathrm{AF} \mathrm{II}}=-0.6 \times 10^{-6} \mathrm{~J} / \mathrm{m}^{2}$, respectively. 


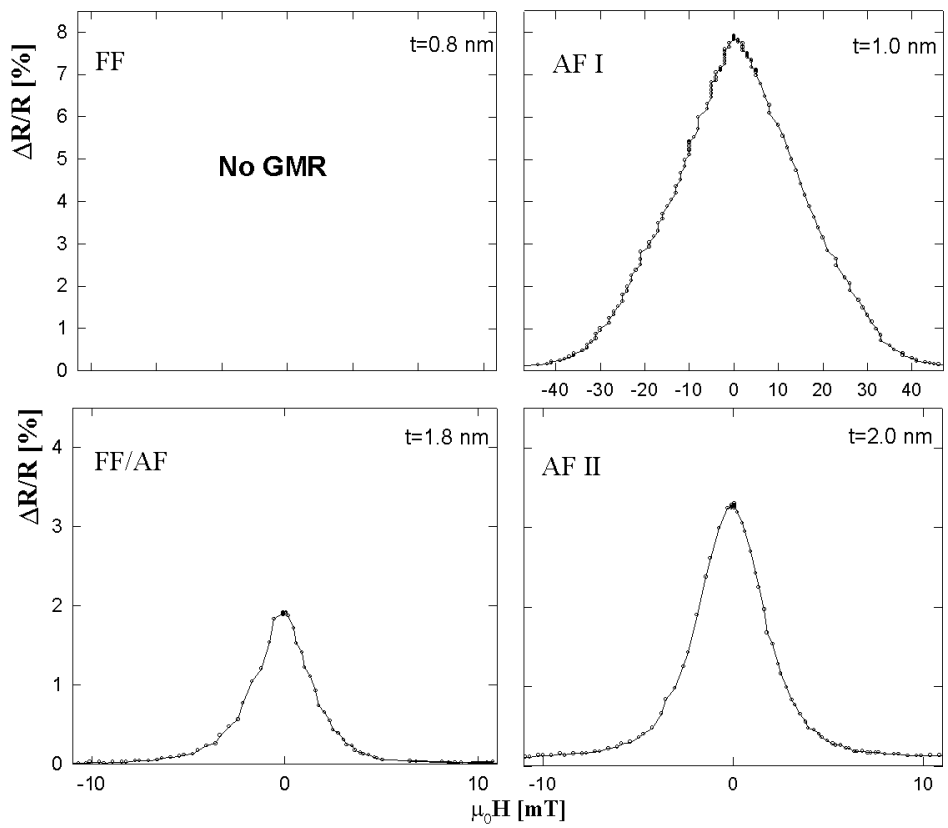

Fig. 4. Magnetoresistivity ratio $(\Delta R / R)$ as a function of external magnetic field of $\mathrm{Ni}_{83} \mathrm{Fe}_{17}(2 \mathrm{~nm}) / \mathrm{Cu}(t)$ for different thickness of $\mathrm{Cu}$ spacer. GMR effect was observed only for AF I, AF II and mixed state FF/AF.

periodic MLs type of $\mathrm{F} /(\mathrm{S} / \mathrm{F})_{N}$ this requirement is fulfilled for antiferromagnetic coupling $(\uparrow \downarrow)$. However, the coupling energy $J$ does not influence the $\Delta R / R$ but determines the $H_{\mathrm{S}}$ value.

Since the discovery of GMR in Fe/Cr MLs [10] this phenomenon was also found in different periodic MLs, where ferromagnetic sublayers are $3 d$ metals ( $\mathrm{Fe}$, $\mathrm{Co}, \mathrm{Ni}$ and their alloys) and predominantly as spacer are used: $\mathrm{Cu}, \mathrm{Ag}, \mathrm{Au}$ and $\mathrm{Ru}$. The outstanding values of GMR $\left(\mathrm{GMR}=\left(R_{\max }-R_{\min }\right) / R_{\min }\right)$ are for $\mathrm{Fe} / \mathrm{Cr}$ $(220 \%$ at $T=1.5 \mathrm{~K})$ [29] and $\mathrm{Co} / \mathrm{Cu}(80 \%$ at RT) [30]. From the application point of view not only high value of GMR is important but also the shape of the dependence of $R(H)$ and value of $H_{\mathrm{S}}$ which determine the field sensitivity of GMR effect. For magnetically isotropic F layers coupled antiferromagnetically in $\mathrm{F} /(\mathrm{S} / \mathrm{F})_{N}$, the $M(H)$ dependence is linear and corresponding $\operatorname{GMR}(H)$ (defined by $\left.\operatorname{GMR}(H)=100 \%\left[R(H)-R\left(H_{\mathrm{S}}\right)\right] / R\left(H_{\mathrm{S}}\right)\right)$ is parabolic according to the relation $\operatorname{GMR}(H) \propto\left[M(H) / M_{\mathrm{S}}\right]^{2}$ for $H<H_{\mathrm{S}}$.

In real MLs multilayer systems apart from antiferromagnetic ordering also perpendicular magnetization alignment appears which gives rise to convexity of $M(H)$ dependence (Fig. 3) and linear (triangle shape) in GMR $(H)$ (Fig. 4). Reversal magnetization process in MLs of NiFe $(2 \mathrm{~nm}) / \mathrm{Cu}\left(t_{\mathrm{Cu}}\right)$ in the form of $M(H)$ and $\operatorname{GMR}(H)$ curves are presented in Fig. 3 for samples with different thickness of Cu spacer. 
The presented dependencies allow us to draw the following conclusions:

- Samples with the thickness of $\mathrm{Cu}$ spacer $t_{\mathrm{Cu}}=1 \mathrm{~nm}$ and $2 \mathrm{~nm}$ show antiferromagnetic coupling (AF I and AF II, respectively (Fig. 3)), which is indicated by $M_{\mathrm{R}} \approx 0$ (antiparallel ordering of magnetizations in neighbouring $\mathrm{F}$ layers) and GMR effect is observed (Fig. 4).

- The saturation field $\left(H_{\mathrm{S}}\right)$ for MLs with $t_{\mathrm{Cu}}=1 \mathrm{~nm}$ (Fig. 3 (AF I )) is about 8 times higher than for $t_{\mathrm{Cu}}=2 \mathrm{~nm}$ (Fig. 3 (AF II)). It is due to the decrease in IEC with increase in the spacer thickness.

- The value of GMR for MLs with $t_{\mathrm{Cu}}=2 \mathrm{~nm}$ (Fig. 4 (AF II)) is smaller than for $t_{\mathrm{Cu}}=1 \mathrm{~nm}$ (Fig. 4 (AF I)) due to the shunting effect (see explanation in Sec. 2).

- The field sensitivity of GMR $\left(S=\right.$ GMR $\left./ H_{\mathrm{S}}\right)$ for MLs with $t_{\mathrm{Cu}}=2 \mathrm{~nm}$ is higher than for $t_{\mathrm{Cu}}=1 \mathrm{~nm}$ because the changes of $H_{\mathrm{S}}$ are bigger than the changes of GMR.

- Ferromagnetically coupled films do not show GMR effect because magnetizations of adjacent layers are always (independently of value of external magnetic field) oriented in parallel.

- Partially ferro- and antiferromagnetically coupled films (for which $0<M_{\mathrm{R}}<$ $M_{\mathrm{S}}$ ) show that GMR is proportional to antiferromagnetically coupled fraction $\left(F_{\mathrm{AF}}\right)$ of MLs according to the relation: $\mathrm{GMR} \propto F_{\mathrm{AF}}=1-M_{\mathrm{R}} / M_{\mathrm{S}}$.

Presented $M(H)$ and GMR $(H)$ curves (Fig. 3 and 4 ) and the properties discussed above are typical also of other $\mathrm{F} /(\mathrm{S} / \mathrm{F})_{N}$ MLs but in numerical values differences of GMR, IEC energy and oscillation period are possible. It should be stated that $\mathrm{NiFe} / \mathrm{Cu}$ MLs achieve for $t_{\mathrm{Cu}}=2 \mathrm{~nm}$ high sensitivity $S=0.6 \% / \mathrm{Oe}$ [21,31-33], although small GMR values (due to small AF coupling energy) are observed. Higher values were obtained for NiFe/Au MLs [34].

\subsection{Spin valves}

The multilayers type of $\mathrm{F}_{1} / \mathrm{S} / \mathrm{F}_{2} / \mathrm{AF}$ is a spin valve ( $\mathrm{SV}$ ) structure $[16,35,36]$ and in its simplest form consists of a "free" ferromagnetically soft layer $\left(\mathbf{F}_{1}\right)$ separated by a non-ferromagnetic metallic spacer layer (S) from the second "pinned" ferromagnetic layer $\left(\mathrm{F}_{2}\right)$, which has its magnetization pinned by a biasing interaction with an antiferromagnetic layer. The spacer layer is thick enough to minimize the magnetic coupling between $\mathrm{F}_{1}$ and $\mathrm{F}_{2}$ ferromagnetic layers.

The exchange biased anisotropy effect was for the first time observed in surface oxidized $\mathrm{Co}$ particles i.e. $\mathrm{CoO} / \mathrm{Co}$ system ( $\mathrm{CoO}$ is an antiferromagnet with Néel temperature $T_{\mathrm{N}}=293 \mathrm{~K}$ [37]). By means of this effect in the system $\mathrm{AF}(\mathrm{CoO}) / \mathrm{F}(\mathrm{Co})$ it is possible to shift hysteresis loop of Co along the field axis with respect to $H=0$. The shift field $H_{\mathrm{EB}}$ is the unidirectional exchange biased 
field expressed by $H_{\mathrm{EB}}=E_{\mathrm{EB}} /\left(M_{\mathrm{F}} t_{\mathrm{F}}\right)$, where $M_{\mathrm{F}}$ and $t_{\mathrm{F}}$ are saturation magnetization and the thickness of ferromagnetic layer, respectively, $E_{\mathrm{EB}}$ is the exchange energy between AF and F layers. The operation of SV can be understood from the magnetization $M(H)$ (Fig. 5a) and magnetoresistance $\Delta R(H)$ curves (Fig. 5b). If the exchange coupling between $\mathrm{F}_{1}$ and $\mathrm{F}_{2}$ is neglected the "free" layer remagnetizes in field $H \approx 0$ whereas the hysteresis loop of the "pinned" layer remagnetizes at $H_{\mathrm{EB}}$. The direction of $H_{\mathrm{EB}}$ field is determined by the external magnetic field direction, during film growth or during temperature decrease from $T>T_{\mathrm{B}}$ to $T_{\mathrm{B}}$ (where $T_{\mathrm{B}}$ is the blocking temperature of frozen spins). For $|H|<\left|H_{\mathrm{EB}}\right|$ the magnetization vectors of $F_{1}$ and $F_{2}$ are oriented antiparallel, therefore in this range of magnetic field a maximum of resistance is observed $\left(R_{\uparrow \downarrow}>R_{\uparrow \uparrow}\right)$ ). It should be noticed that $E_{\mathrm{EB}}$ and $H_{\mathrm{EB}}$ decrease with temperature increasing and vanish at $T \geq T_{\mathrm{B}}$. Therefore proper selection of antiferromagnetic materials with regard to temperature stability of spin valve is very important.

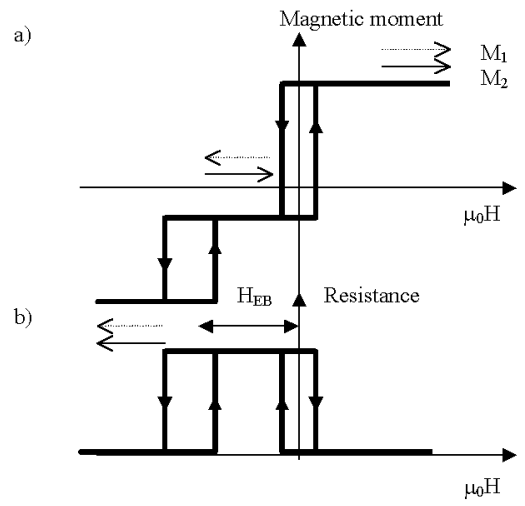

Fig. 5. Schematic presentation: (a) magnetization and (b) magnetoresistivity hysteresis loop of the spin valve structure. $M_{1}, M_{2}$ are the magnetizations of free and pinned layer, respectively.

Some representative values of $E_{\mathrm{EB}}, \mathrm{T}_{\mathrm{N}}$, and $T_{\mathrm{B}}$ are given in Table. AF oxides (e.g. NiO) due to high resistivity in opposite to metallic AF alloy (e.g. $\mathrm{FeMn}$ ) do not show the electrical shunting of $\mathrm{F}_{1} / \mathrm{S} / \mathrm{F}_{2}$ segment of $\mathrm{SV}$. The crucial point for GMR enhancement is the spacer thickness. As it was mentioned in Sec. 2 too thick spacer layer decreases GMR, however too thin gives rise to increase in the probability of creation of pinholes between $\mathrm{F}_{1}$ and $\mathrm{F}_{2}[17,20]$ and also increases the influence of magnetostatic interactions due to interface roughness [38-41]. When interlayer exchange energy $\left(J_{\mathrm{F}}\right)$ increases with respect to the energy exchange biased $\left(E_{\mathrm{EB}}\right), j=J_{\mathrm{F}} / E_{\mathrm{EB}}$, then the difference of remagnetizing fields of "free" $\left(\mathrm{F}_{1}\right)$ and "pinned" $\left(\mathrm{F}_{2}\right)$ layers gradually vanishes. It takes place at $j>j_{\text {crit }}$ (where $j_{\text {crit }}=0.25$ for "free" and "pinned" layers from the same materials and equal thickness). In this case the magnetization rever- 
TABLE

Typical antiferromagnetic materials in the spin valve structure. $E_{\mathrm{EB}}-$ exchange biased energy, $T_{\mathrm{N}}-$ Néel temperature, $T_{\mathrm{B}}$ - spin blocking temperature [27]. "poly-ann" means polycrystalline after annealing.

\begin{tabular}{l|c|c|c}
\hline \hline \multicolumn{1}{c|}{$\mathrm{AF}$ materials } & $E_{\mathrm{EB}}\left[\mathrm{mJ} / \mathrm{m}^{2}\right]$ & $T_{\mathrm{N}}[\mathrm{K}]$ & $T_{\mathrm{B}}[\mathrm{K}]$ \\
\hline $\mathrm{Fe}_{50} \mathrm{Mn}_{50}$ (poly-ann.) & $0.05-0.47$ & 490 & 423 \\
$\mathrm{Ni}_{50} \mathrm{Mn}_{50}$ (poly-ann.) & $0.16-0.47$ & 1070 & 770 \\
$\mathrm{Pt}_{50} \mathrm{Mn}_{50}$ (poly-ann.) & $\leq 0.32$ & 480 & 400 \\
$\mathrm{Ir}_{18} \mathrm{Mn}_{82}$ (poly-ann.) & 0.19 & 690 & 538 \\
$\mathrm{NiO}$ & $0.05-0.29$ & 535 & 453 \\
$\mathrm{CoO}$ & $0.14-0.48$ & 293 & $\leq 293$
\end{tabular}

sal of "free" layer is simultaneously accompanied with magnetization process of "pinned" layer and the antiparallel alignment of magnetizations of adjacent layers is impossible. As a consequence with increasing $J$, for $j>j_{\text {crit }}$ GMR decreases. In order to compensate the influence of ferromagnetic coupling the thickness of spacer layer should be optimized. The highest GMR sensitivity values were obtained in the range of $t_{\mathrm{S}}$ assuring weak antiferromagnetic interlayer exchange coupling. For $\mathrm{Cu}$ as spacer layer the optimal thickness is $20 \AA$ to $22 \AA$ [39]. It is clear that also the state of the crystal structure of $\mathrm{Cu}$ is influenced by deposition methods and conditions [42] and consequently the structure imperfections modify the effective coupling between $\mathrm{F}_{1}$ and $\mathrm{F}_{2}$ layers. Figure 6 presents $M(H)$ and $\operatorname{GMR}(H)$ curves for two identical (with respect to applied material and thickness of particular layers) spin valve structures deposited by sputtering technique: $\mathrm{Si}(100) / \mathrm{SiO} / \mathrm{Ta}_{1}(52 \AA) / \mathrm{Co}_{1}(44 \AA) / \mathrm{Cu}_{1}(22 \AA) / \mathrm{Co}_{2}(44 \AA) / \mathrm{FeMn}(85 \AA) /$ $\mathrm{Ta}_{2}(52 \AA) / \mathrm{Cu}_{2}(5 \AA)$ (where segments: $\mathrm{Si}(100) / \mathrm{SiO} / \mathrm{Ta}_{1}(52 \AA)$ are substrate and buffer layers, $\mathrm{FeMn}(85 \AA)$ is an antiferromagnet and $\mathrm{Ta}_{2}(52 \AA) / \mathrm{Cu}_{2}(5 \AA)$ are capping layers which adjust the contact to electrodes and protect for oxidation). The $\mathrm{SV} 1$ is characterized by stronger ferromagnetic coupling than SV $2\left(J_{\mathrm{F}_{1}}>J_{\mathrm{F}_{2}}\right)$, hence difference in switching fields of free and pinned layers is smaller for SV 1 than for SV 2 and in consequence GMR is larger for SV 2 than for SV 1 (more details can be found in paper [41]).

The spin valves are characterized by very high GMR sensitivity up to $17 \% / \mathrm{Oe}$ [43], therefore are used as read element of heads for high density hard disc drive (HDD) since 1999. The GMR-SV system provides many advantages over AMR-heads: excellent signal (as high as $3.6 \mathrm{mV}_{\mathrm{p}-\mathrm{p}}$ ) and enhanced thermal stability [44].

Willekens et al. [45] proposed in the classical SV replacing the segment $\mathrm{F}_{2} / \mathrm{AF}$ by three layers segment $\mathrm{F}_{2} / \mathrm{S}_{2} / \mathrm{F}_{3}$ where $\mathrm{F}_{2}$ and $\mathrm{F}_{3}$ are in the state of strong antiferromagnetic interlayer exchange coupling. Such type of SV $\left(F_{1} / S_{1} / F_{2} / S_{2} / F_{3}\right)$ is called as AF-biased spin valve (segment $\mathrm{F}_{2} / \mathrm{S}_{2} / \mathrm{F}_{3}$ is known in literature as syn- 

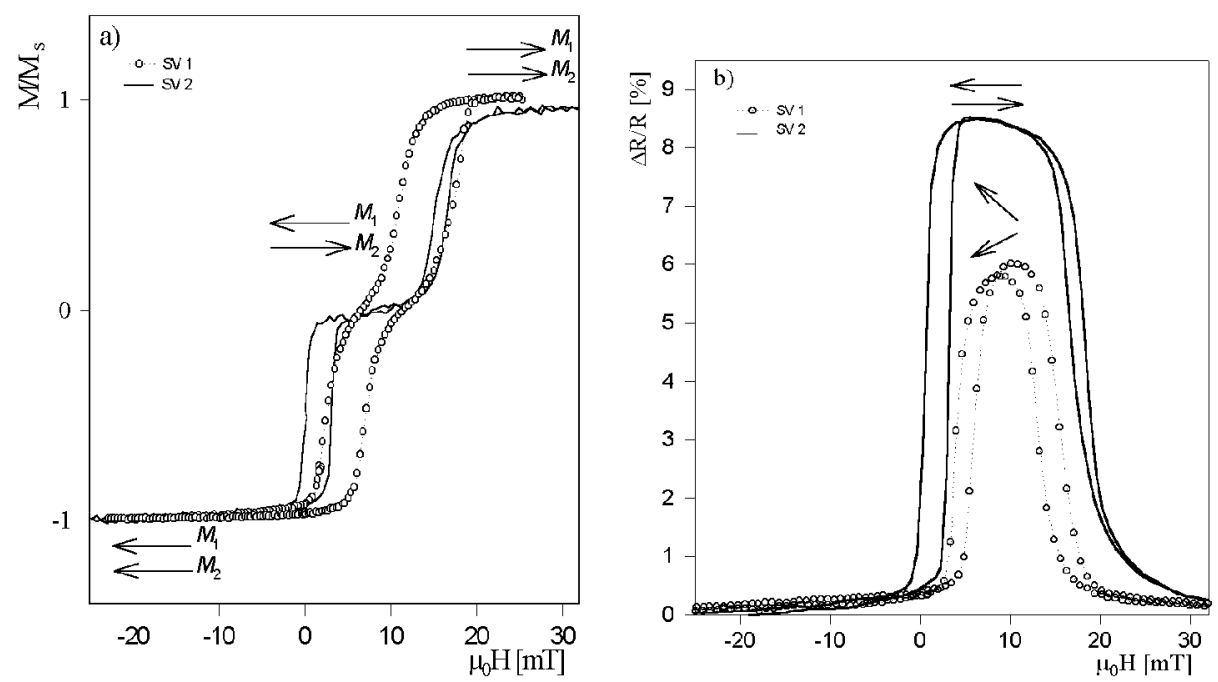

Fig. 6. Magnetization (a) and magnetoresistivity (b) hysteresis loops of two spin valve structures SV 1 and $\mathrm{SV} 2 . \mathrm{SV} 1-\mathrm{Cu}_{1}$ spacer sputtered a.t $2.4 \mathrm{~kW} R \mathrm{RF}, J_{\mathrm{F}}=$ $0.033 \mathrm{~mJ} / \mathrm{m}^{2}, E_{\mathrm{EB}}=0.12 \mathrm{~mJ} / \mathrm{m}^{2}, j=0.28 \mu_{0} H_{\mathrm{EB}}=16 \mathrm{mT}$. SV $2-\mathrm{Cu}_{1}$ spacer sputtered at $1.5 \mathrm{~kW} \mathrm{DC}, J_{\mathrm{F}}=0.012 \mathrm{~mJ} / \mathrm{m}^{2}, E_{\mathrm{EB}}=0.11 \mathrm{~mJ} / \mathrm{m}^{2}, j=0.11, \mu_{0} H_{\mathrm{EB}}=14 \mathrm{mT}$. $\mathrm{Si}(100) / \mathrm{SiO} / \mathrm{Ta}_{1}(52 \AA) / \mathrm{Co}_{1}(44 \AA) / \mathrm{Cu}_{1}(22 \AA) / \mathrm{Co}_{2}(44 \AA) / \mathrm{FeMn}(85 \AA) / \mathrm{Ta}_{2}(52 \AA) /$ $\mathrm{Cu}_{2}(5 \AA)$.

thetic antiferromagnet (SAF)) and is used as very precise angle position sensor (e.g. the steering wheel in cars or the program selector of washing machines) [46].

\subsection{Pseudo-spin valves}

Other solution for obtaining the transition, under external magnetic field, from parallel to antiparallel configuration of magnetizations in two ferromagnetic layers are structures of the type $\mathrm{F}_{1} / \mathrm{S} / \mathrm{F}_{2}$ in which $\mathrm{F}_{1}$ and $\mathrm{F}_{2}$ are ferromagnetic layers of different coercivity (for example $\mathrm{Ni}_{80} \mathrm{Fe}_{20} \mathrm{Co}$ (soft), $\mathrm{CoFe}$ (hard $H_{\mathrm{C}_{2}}>H_{\mathrm{C}_{1}}$ )) separated by non-ferromagnetic layer (for example $\mathrm{Cu}$ ) [47-49]. Due to thick $\mathrm{Cu}$ spacer $(\geq 20 \AA)$ the exchange coupling between soft $\left(\mathrm{F}_{1}\right)$ and hard $\left(\mathrm{F}_{2}\right)$ is neglected. Such structure is known as pseudo-spin valve (PSV). The typical magnetization and magnetoresistivity hysteresis loops are presented in Fig. 7. From the application point of view the large difference of $H_{\mathrm{C}_{1}}$ and $H_{\mathrm{C}_{2}}$ is desirable (Fig. 7a). In PSV like in SV the ferromagnetic coupling (magnetostatic or caused by pinholes) reduces the difference between coercivity fields of both ferromagnetic layers and GMR values.

Due to specific behaviour of $\Delta R / R(H)$ dependence (Fig. 7b), correlated with magnetization reversal process, PSV can be used as cells of magnetoresistive random access memories (M-RAM) $[6,7,50]$, due to the difference between resistance 

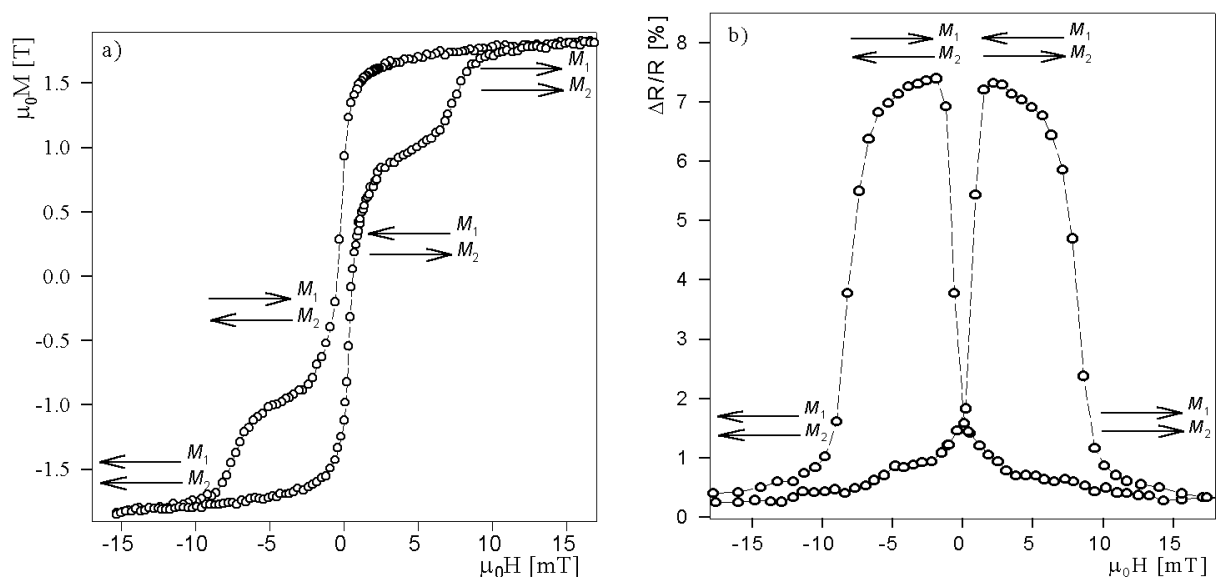

Fig. 7. (a) Magnetization and (b) magnetoresistivity hysteresis loop of pseudo-spin valve consisting of $\mathrm{Ni}_{80} \mathrm{Fe}_{20}(2.8 \mathrm{~nm}) / \mathrm{Co}(2.1 \mathrm{~nm}) / \mathrm{Cu}(2 \mathrm{~nm}) / \mathrm{Co}(3 \mathrm{~nm}) . M_{1}, M_{2}$ denote the magnetization of hard $(\mathrm{Co})$ and soft $\left(\mathrm{Ni}_{80} \mathrm{Fe}_{20}\right)$ layer, respectively.

values of ferromagnetic (low resistivity) and antiferromagnetic (high resistivity) alignment of magnetization vectors.

The main advantages of M-RAM over the currently used dynamic RAM's are: simple memory cells construction and preparation, non-volatile, unlimited read and write endurance, high speed operation.

\section{Summary}

The main requirement to obtain GMR in layered structures is reorientation of mutual magnetization directions in neighbouring ferromagnetic layers induced by magnetic field. This requirement can be fulfilled in different artificial structures consisting of two (or more) ferromagnetic layers separated by non-ferromagnetic layers (see Sec. 3). This enables the realization of elements with attractive magnetoresistive characteristics for particular applications. However, considerable GMR values can be obtained only for thicknesses of individual layers smaller than $3 \mathrm{~nm}$. For such thin film structures, magnetic interlayer coupling as well as GMR effect are strongly dependent on the roughness of interfaces and other structural imperfections such as pinholes or mixed interfaces.

\section{Acknowledgment}

The authors are grateful to the State Committee for Scientific Research for financial support under grants: 8T11B 05418 and PBZ/KBN-013/T08/23.

\section{References}

[1] C. Kittel, Quantum Theory of Solids, Wiley, New York 1963, Ch. 12, p. 237.

[2] T.R. Mc Guire, R.I. Potter, IEEE Trans. Magn. 11, 1018 (1975). 
[3] E.N. Mitchell, H.B. Haukaas, H.D. Bale, J.B. Streeper, J. Appl. Phys. 35, 2604 (1964).

[4] Z. Szczaniecki, F. Stobiecki, R. Gontarz, H. Ratajczak, Phys. Status Solidi A 18, 411 (1973).

[5] T. Stobiecki, A. Paja, Acta Phys. Pol. A 41, 343 (1972).

[6] S. Tumański, Thin Film Magnetoresistive Sensors, Institute of Physics Publishing, Bristol 2001.

[7] H.K. Lachowicz, J. Techn. Phys. 42, 127 (2001).

[8] J.S. Moodera, L.R. Kinder, T.M. Wong, R. Meservey, Phys. Rev. Lett. 74, 3273 (1995).

[9] R.v. Helmholt, J. Wecker, B. Holzapfel, L. Schultz, K. Samwer, Rev. Lett. 71, 2331 (1993).

[10] M.N. Baibich, J.M. Broto, A. Fert, Nguen Van Dau, F. Petroff, P. Etienne, G. Creuzet, A. Friederich, J. Chazelas, Phys. Rev. Lett. 61, 2472 (1988).

[11] G. Binash, P. Grünberg, F. Sauernbach, W. Zinn, Phys. Rev. B 39, 4828 (1989).

[12] P. Grünberg, R. Schreiber, Y. Pang, M.B. Brodsky, H. Sowers, Phys. Rev. Lett. 57, $2442(1986)$

[13] C. Carbone, S.F. Alvarado, Phys. Rev. B 36, 2433 (1987).

[14] J. Mathon, Contemp. Phys. 32, 143 (1991).

[15] N.F. Mott, Adv. Phys. 13, 325 (1964).

[16] B. Dieny, V.S. Speriosu, S.S.P. Parkin, B.A. Gurney, D.R. Wilhoit, D. Mauri, Phys. Rev. B 43, 1297 (1991).

[17] J.F. Bobo, M. Piecuch, E. Snoeck, J. Magn. Magn. Mater. 126, 440 (1993).

[18] H. Kikuchi, J.F. Bobo, L.R. White, IEEE Trans. Magn. 33, 3583 (1997).

[19] F. Stobiecki, T. Luciński, J. Dubowik, B. Szymański, M. Urbaniak, F.J. Castaño, T. Stobiecki, J. Magn. 3, 89 (1998).

[20] F. Stobiecki, T. Luciński, R. Gontarz, M. Urbaniak, Mater. Sci. Forum 287-288, 513 (1998).

[21] T. Luciński, F. Stobiecki, D. Elefant, D. Eckert, G. Reiss, B. Szymański, J. Dubowik, M. Schmidt, H. Rohrmann, K. Röll, J. Magn. Magn. Mater. 174, 192 (1997).

[22] S.S.P. Parkin, in: Ultrathin Magnetic Structures, Vol. II, Eds. J.A.C. Bland, B. Heinrich, Springer-Verlag, Berlin 1994.

[23] A. Fert, P. Bruno, in: Ultrathin Magnetic Structures, Vol. II, Eds. J.A.C. Bland, B. Heinrich, Springer-Verlag, Berlin 1994.

[24] P. Bruno, in: Magnetische Schichtsysteme, Eds. P. Grünberg, P.H. Dederichs, Forschungszentrum, Jülich 1999, p. B8.1.

[25] P. Grünberg, in: Magnetische Schichtsysteme, Eds. P. Grünberg, P.H. Dederichs, Forschungszentrum, Jülich 1999, p. B9.1.

[26] D.E. Bürgler, in: Magnetische Schichtsysteme, Eds. P. Grünberg, P.H. Dederichs, Forschungszentrum, Jülich 1999, p. B10.1.

[27] D.E. Bürgler, P. Grünberg, in: Neue Materialen für die Informationstechnik, Ed. R. Waser, Forschungszentrum, Jülich 2001, p. A4.1. 
[28] P.A. Grünberg, Sensors Actuators A 91, 153 (2001).

[29] R. Schad, C.D. Potter, P. Beliën, G. Verbanck, V. V. Moshchalkov, Y. Brunseraede, Appl. Phys. Lett. 64, 3500 (1994).

[30] H. Kano, K. Kagawa, A. Suzuki, A. Okabe, H. Hayashi, K. Aso, Appl. Phys. Lett. 63, 2839 (1993)

[31] S.S.P. Parkin, Appl. Phys. Lett. 60, 512 (1992).

[32] T. Luciński, F. Stobiecki, B. Szymański, J. Dubowik, M. Schmidt, M. Urbaniak, J. Phys. IV (France) 8, Pr-2-453 (1998).

[33] T. Stobiecki, F. Stobiecki, M. Czapkiewicz, J. Wrona, F.J. Castaño, J.L. Muñoz, J. Magn. Magn. Mater. 196-197, 107 (1999).

[34] S.S.P. Parkin, T. Rabedeau, Appl. Phys. Lett.68, 1162 (1996).

[35] B. Dieni, J. Magn. Magn. Mater. 136, 335 (1994).

[36] J. Nognés, I.K. Schuler, J. Magn. Magn. Mater. 192, 203 (1999).

[37] W.H. Meiklejohn, C.P. Bean, Phys. Rev. 102, 1413 (1956); 105, 904 (1957).

[38] J. Zhang, R.M. White, J. Appl. Phys. 79, 5113 (1996).

[39] Th.G.S.M. Rijks, R. Coehoorn, J.T.F. Daemen, W.J.M. de Jonge, J. Appl. Phys. 76, 1092 (1994).

[40] J.L. Leal, M.H. Kryder, IEEE Trans. Magn. 32, 4642 (1996).

[41] F. Stobiecki, T. Stobiecki, B. Ocker, W. Maass, W. Powroźnik, A. Paja, C. Loch, K. Röll, Acta Phys. Pol. A 97, 523 (2000).

[42] W.F. Egelhoff, Jr., P.J. Chen, C.J. Powell, D. Parks, R.D. McMichael, J.H. Judy, D. Martien, A.E. Berkowitz, J.M. Daughton, in: Proc. Int. Nonvolatile Memory Technology Conf., Albuquerque 1998, IEEE, 1998, p. 34.

[43] C.-M. Park, H. Shin, Appl. Phys. Lett. 70, 776 (1997).

[44] H.C. Tong, X. Shi, F. Liu, C. Qian, Z.W. Dong, X. Yan, R. Barr, L. Miloslavsky, S. Zhou, J. Perlas, P. Prabhu, M. Mao, S. Funada, M. Gibbson, Q. Leng, J.G. Zhu, S. Dey, IEEE Trans. Magn. 35, 2574 (1999).

[45] M.M.H. Willekens, Th.G.S.M. Rijks, H.J.M. Swagten, W.J.M. de Jonge, J. Appl. Phys. 78, 7202 (1995).

[46] H.A.M. van der Berg, W. Clemens, G. Gieres, G. Rupp, W. Schelter, M. Vieth, IEEE Trans. Magn. 32, 4624 (1996).

[47] T. Shinjo, H. Yamamoto, J. Phys. Soc. Jap. 59, 3061 (1990).

[48] B. Dieny, V.S. Speriosu, B. A. Gurney, S.S.P. Parkin, D.R. Wilhoit, K.P. Rosche, S. Metin, D.T. Peterson, S. Nadimi, J. Magn. Magn. Mater. 93, 101 (1991).

[49] W.E. Egelhoff, P.J. Chen, C.J. Powell, M.D. Stiles, R.D. McMichael, C.-L. Lin, J.M. Sivertsen, J.H. Judy, K. Takano, A.E. Berkowitz, T.C. Anthony, J.A. Brug, J. Appl. Phys. 79, 5277 (1996).

[50] M. Czapkiewicz, T. Stobiecki, J. Wrona, W. Machowski, in: Proc. 7th Int. Conf. Mixed Design of Integrated Circuits and Systems, MIXDES 2000, Gdynia (Poland) 2000, Ed. A. Napieralski, Technical University of Łódź, Łódź 2000, p. 297. 